\title{
The Probe Into Unpitched Sound Generator Design Based On Masking Effect And Audio Alarm
}

\author{
Dian $\mathrm{Gu}^{1 \mathrm{a}}$, Dongsong Yan ${ }^{1}$, Junhao Liu ${ }^{2}$ \\ Jinan University/Rail Transportation Research Institute ${ }^{1}$ 519070, Shenzhen Energy Environment \\ Engineering Co., Ltd $^{2} 518000$
}

Keywords: unpitched sound generator, masking effect, MCU, audio alarm

Abstract. Noise pollution does harm to urban residents, so we design an unpitched sound generator using masking effect in psychoacoustics, which can conceal or compete with ambient noise, in order to help people to be more concentrate. It can also generate warning noise according to the speed of electric bikes to improve driving safety. Experiment shows that natural noise has the best masking effect, while Meaningless noise has the highest masking efficiency. This sound generator we design is extensible and easy-to-use, and has high value, providing methods approach to compete noise interference and use noise to enhance security for a practical application.

\section{Introduction}

It's well known that noise does harm to our health. Noise damages auditory functions, affects language communication, divert attention, disturb rest and sleep, etc. To solve noise pollution, we should use administrative means together with technological means. To achieve the effect of noise masking, we can use the way of energetic masking, involving the treating processes of peripheral nervous system. Energetic masking affects the explanation of sound propagation based on physics mode. It uses unpitched sound generator to generate natural noise or pink noise, suppresses noise interference and reduces the sensitivity of the ear to the noise. This method can eliminate the harmful effect of noise, and make people more concentrate.

Besides, with the improvement of automated processes, the number of electric bikes is increasing. However, the noise that electric bikes make is very small, and can't catch pedestrians' attention on the noisy road, which poses a safety hazard to pedestrians. So it's necessary to add traffic noise for high-speed electric bikes. We can alarm pedestrians by playing corresponding noise according to the speed, in order to ensure the safety of vehicles and pedestrians and reduce the incidence of traffic accidents.

In face of noise pollution and safety issues of electric bikes, we suggest a noise generator based on MCU using masking effect in psychoacoustics, which generates specific stable noise to conceal or compete with ambient noise in order to help people to be more concentrate; and generates warning noise according to the speed of electric bikes to improve driving safety.

\section{System designing}

This system generates many kinds of appropriate Masking noise signals and warning sound signals. Those signals are played by audio playback function. Because there are several kinds of electric bikes and the speed range is huge, we match different speeds with different alarm noises. Using Hall sensor module to measure the speed of vehicle wheels, this system can calculate wheel speed and implement audio selection operation according to it.

Function selection and parameter setting are controlled by simple key operations. In view of portability, we provide rechargeable DC power module. The system structure diagram is shown in Fig.1. 


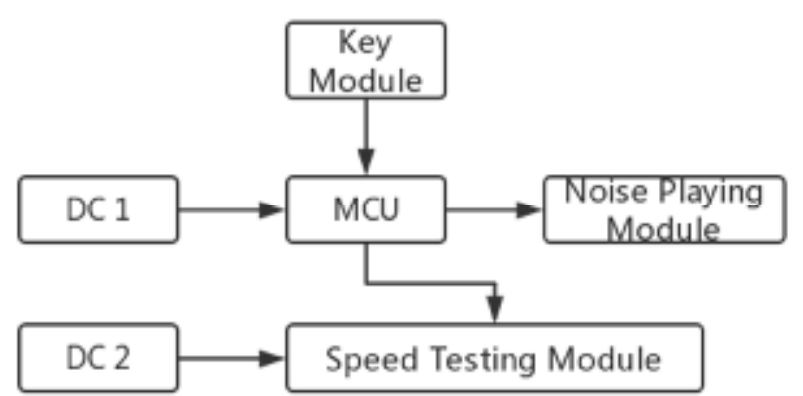

Fig.1 System structure diagram of Unpitched Sound Generator

The system imports designed noise signals into TF card outside. MCU decodes the signals and plays the noise by the loudspeaker, to implement the function of masking environment noise. At the same time, users can choose functions they want including volume adjustment, noise type switchover, and MP3 function by inputting key signals.

In the design of sound alarm system of electric bikes, first we process simulated noise of electric bikes and import it into TF card outside. Second, we generate warning noise according to the speed of electric bike to alarm pedestrians. The warning noise stops when the bike stops. Besides, users can choose different kinds of warning noise signals by inputting key signals. When encountered special circumstances, users can play shrill whistle to raise alert effect.

\section{Selection of noise signal source}

Firstly, the Selection of noise signal source should put the masking effect on the first place, while the masking efficiency is on the second place; Secondly, the evaluation of noise signals should take people's well-being and eco-demand into consideration, so different kinds of noises are necessary to satisfy different needs; Thirdly, noise signals should adjust its signal waveform according to frequency response of loudspeaker to have the best effect; Finally, we should consider warning effect when the noise signals are used for alarm function.

This system divide noises into two types: masking sound signals and warning sound signals. The former includes meaningless noise generated by Matlab functions, natural noise cut from CD released by Cobra Entertainment LLC and artificial analog noise recorded by ourselves; the latter simulates the sound of bike, motorbike and emergency noise.

\section{System implement}

\section{Hardware design}

The hardware of this system includes high-speed MCU, MP3 decoding chip, 16GB TF card, EXT SPKR, NJK-5002B HS. The HS is installed on the saddle and fixed by the steel hoop, and there is a permanent magnet stick on the spoke. The HS is powered up by Separate dc power supply. During the ride, HS has a digital signal when the vehicle wheel makes a round, and the MCU processes this signal to get the wheel rotation period, and then calculate the wheel speed.

\section{Software design}

This system is programed by $\mathrm{C}$ language, and it's divided into two parts: the main program and four submodules. The submodules include MP3(audio playback module), USART1(speed measurement module), BUTTON(key module) and SPEED(speed measuring module).

The main program invokes functions from submodules directly to realize functions like initialization, input value reading, play control and file selection. The audio playback module includes functions like volume control, play control and file selection. The speed measurement module includes three programs: the initialization of timer 0 and interrupt mark bit; timer 0 interrupt program and external interrupt 0 interrupt program. The main function of key module is to read the key signal from IO and to do the shake elimination processing, and then returns the corresponding flag value for each key. The algorithm flow chart of key module is shown in Fig.2. 


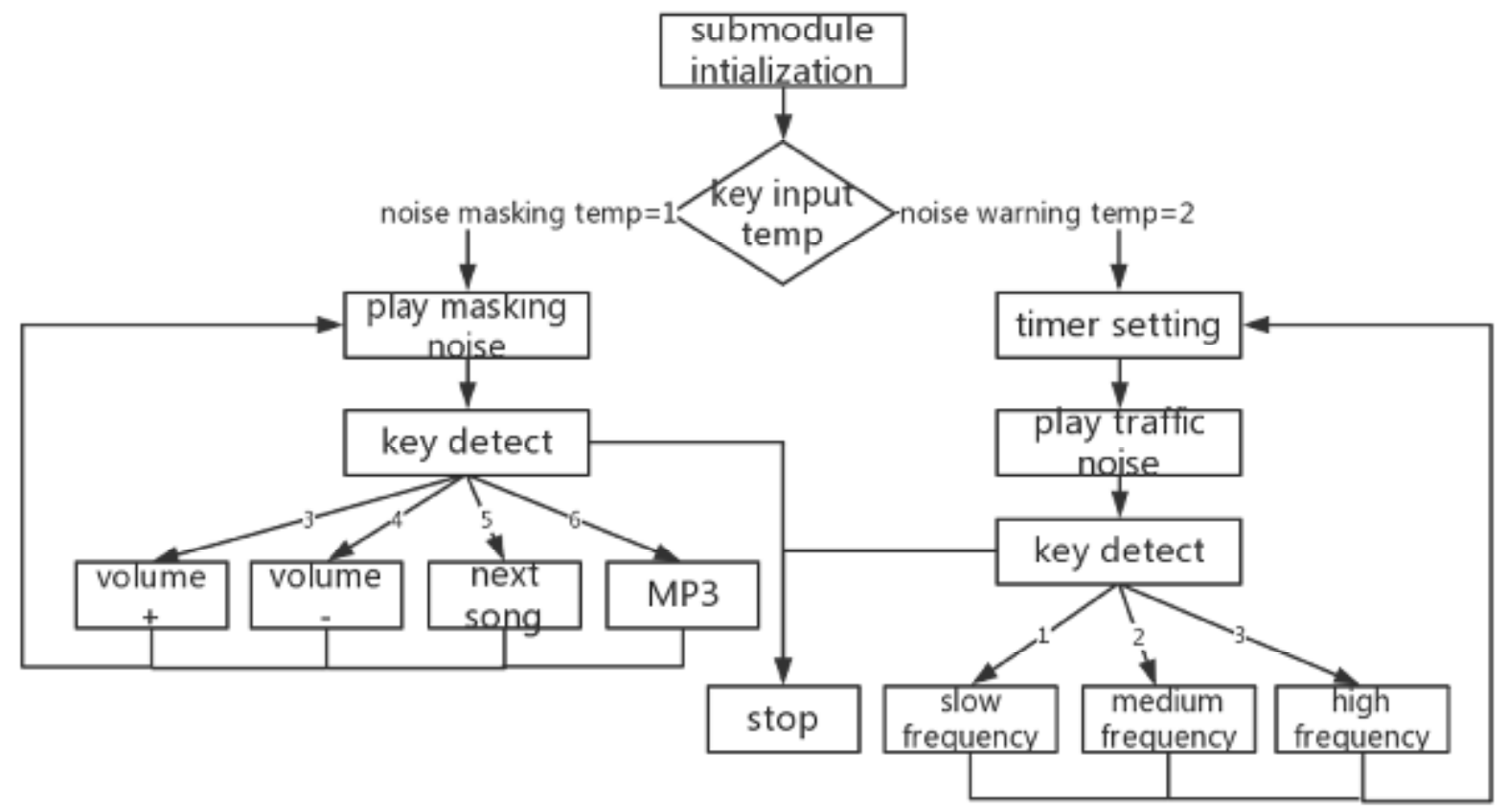

Fig.2 algorithm flow chart of key module

\section{System operation test}

\section{Masking noise effect evaluation}

We did objective evaluation and subjective evaluation for masking noise. Subjective evaluation: record the noise of piling construction site, and the spectrogram is shown in Fig.3. Then we opened the noise generator, and chose the masking noise playing function, played the pink noise. When the pile driver opened again, we recorded the construction site again, and the spectrogram is shown in Fig.4.

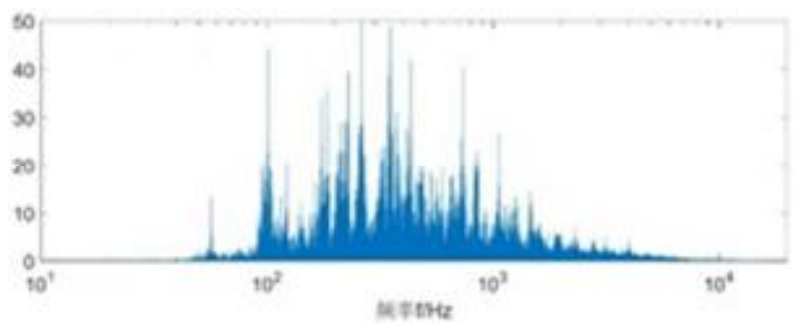

Fig.3 environmental noise spectrum without masking noise

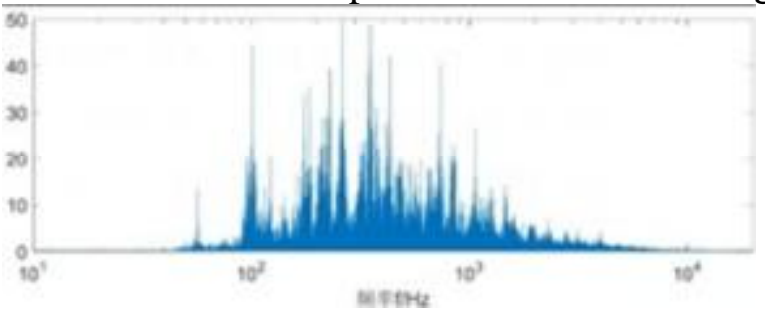

Fig.4 environmental noise spectrum with masking noise

After comparison, we found that the power of environmental noise is focused on low frequency. After adding masking noise, the pulse value of environmental noise reduced obviously, and the power of environmental noise is focused on low frequency and medium frequency. So there are some masking effects on environmental noise pulses when using masking noise.

Objective evaluation: the experimental environment is in the living quarters 20 meters far from noisy construction site. The noise source mainly includes the sound of car driving, the sound of excavator work and the regular pulsing of pile machine. The speaker and the experimental equipment noise mask are placed 1 meter ahead of the tester. Four men, aged 21 to 24 , are asked to 
test a tester in an experimental environment. They evaluate three indexes of the environmental noise and masking noise in two minutes. Then start the noise generator to play the masking noise. After each noise is played for 20 to 30 seconds, the three indexes are evaluated again. The experimental results show that after adding masking noise, the noise level of the human feeling is reduced, the speech clarity becomes blurred, the human body reduces the sensitivity to environmental noise. It is shown that masking noise has a good masking effect on environment noise and speech. When the noise device is placed in front of the user or directly above, and it is broadcast to the user, the screen has the highest efficient.

More experimental results also indicate that meaningless and steady-state natural noise masking efficiency is higher, but the masking effect is poorer. Pulse natural noise makes people happier, but the masking efficiency is low. In a noisy environment, HVAC noise on masking efficiency and masking effect are both well.

\section{Electric bike warning noise effect evaluation}

The MCU will automatically play the corresponding alarm noise according to the measured traveling speed, and the sound pressure level of the noise at different speeds is different. Obviously, electric bicycles with noise are more alarming than near-noiseless electric bikes, and the number of people responding to the alarm is significantly larger. But result shows that the spreading distance of simulated motorcycle noise is longer than simulated bicycle noise, and its sound pressure level is higher, so its warning effect is better; After opening the noise alarming system, the alertness of pedestrians and non-motor vehicles in the lane improved effectively. It's useful for pedestrians to judge the speed of the electric bicycle nearby, so as to take the corresponding dodging measures in advance.

\section{Summary}

In view of the increasingly serious noise pollution problem and electric bicycle driving safety problem, based on the psychoacoustic masking effect as theoretical basis, we design the noise generator based on MCU which can achieve noise masking function and electric bicycle sound warning function. With simple portability, various masking noise sources can be provided to obtain better masking effect, and the adaptability to the environment is higher. Experimental tests show that the designed noise generator has good noise masking function, and can effectively reduce the environmental noise interference of the harm to human body, to better protect people's physical and mental health. Its sound warning function can obviously improve the pedestrian's alertness to electric bicycle, so it's of great value to improve the safety of electric bicycle and to better maintain the road safety and is worthily popularized.

\section{References}

[1] Zhonglin Pan, Guoqing Zhai: Noise Control Technology in Chinese. [M]. Chemical Industry Press (2006).

[2] Hao Xie: The Masking Effect of Sound and its Application in Chinese. [J]. Industrial Construction (1998, 28(3): 1-2. ).

[3] Li Wang, Jianling Hu, Sheng Xu: The Study of Speech Enhancement Algorithm Based on Auditory Masking Effect in Chinese. [J]. Audio Engineering (2006(7): 39-42.).

[4] Tianpei Feng, Yansong Wang, Hui Guo: The In-car Noise Auditory Time Domain Masking Active Control LMS Algorithm in Chinese. [D]. Noise and Vibration Control (2014, 34(3): 97-100.). 